\title{
Lesões bucais diagnosticadas na campanha de prevenção do câncer bucal no município
} de Fernandópolis/SP no ano de 2017

Oral lesions diagnosed in the 2017's oral cancer prevention campaign in the city of Fermandópolis/SP Lesiones orales diagnosticadas en la campaña de prevención del cáncer oral en la ciudad de Fernandópolis/SP en 2017

Caroline Liberato Marchiolli

ORCID: https://orcid.org/0000-0001-8881-4882 Universidade Brasil, Brasil

E-mail: caaliberato@gmail.com

Renan Lemos da Silva

ORCID: https://orcid.org/0000-0001-5837-410X Universidade Brasil, Brasil

E-mail: renan15998@gmail.com

Maria Eduarda de Freitas Santana Oliveira ORCID: https://orcid.org/0000-0002-4074-4288 Universidade Brasil, Brasil

E-mail: mariaefreitas10@gmail.com

Luana Ferreira Oliveira

ORCID: https://orcid.org/0000-0002-6478-7119 Universidade Brasil, Brasil

E-mail: luana.oliveiraf@hotmail.com

Vitória Parmejane de Oliveira

ORCID: https://orcid.org/0000-0001-5867-8488 Universidade Brasil, Brasil

E-mail: vitória.parmejan@gmail.com

Saygo Tomo

ORCID: https://orcid.org/0000-0002-2295-7968 Universidade Estadual Paulista"Júlio de Mesquita Filho", Brasil

E-mail: saygotomo@hotmail.com

Paulo Sergio Morais Sales

ORCID: https://orcid.org/0000-0003-1017-8665 Universidade Brasil, Brasil

E-mail: paulomsales@hotmail.com

Karina Gonzalez Camara Fernandes ORCID: https://orcid.org/0000-0003-4644-3408 Universidade Brasil, Brasil

E-mail: karinagcf@yahoo.com.br

Luciana Estevam Simonato

ORCID: https://orcid.org/0000-0002-6413-5479 Universidade Brasil, Brasil

E-mail: lucianasimonato@gmail.com

\section{Resumo}

Introdução: A Odontologia tem um grande desafio frente ao combate do câncer de boca, sendo este considerado um problema de saúde pública. Dessa forma, é importante que a população seja orientada quanto aos fatores de risco para essa doença e como preveni-la. O objetivo deste trabalho é apresentar as lesões bucais diagnosticadas na campanha de prevenção do câncer bucal no município de Fernandópolis/SP no ano de 2017. Metodologia: Inicialmente, os pacientes foram examinados pelos cirurgiões-dentistas das unidades básicas de saúde e, frente a presença de lesões bucais, foram encaminhados para o Centro de Especialidades Odontológicas (CEO) do município para reavaliação por um especialista. Resultados: Foram examinadas 720 pessoas durante a campanha de prevenção do câncer bucal no município de Fernandópolis/SP no ano de 2017, sendo que 58 (8,05\%) dessas apresentaram lesões bucais e foram encaminhadas para reavaliação no CEO. Apenas 52 compareceram a consulta agendada, ou seja, 6 (10,3\%) pessoas evadiram. Dos pacientes reavaliados, $3(5,8 \%)$ apresentaram lesões de caráter maligno e precisaram ser encaminhados para tratamento especializado. Os demais pacientes reavaliados apresentaram 47 lesões benignas ou potencialmente malignizáveis e 19 variações da normalidade foram diagnosticadas. Conclusões: Diante dos resultados obtidos, pudemos concluir que a campanha de prevenção do câncer de boca proporcionou o diagnóstico de um número grande de lesões bucais mesmo 
não tendo a dimensão necessária, pois apenas uma pequena parcela da população do município foi avaliada durante a campanha.

Palavras-chave: Saúde coletiva; Câncer bucal; Odontologia.

\begin{abstract}
Introduction: Dentistry has a great challenge in the fight against oral cancer, which is considered a public health problem. Thus, it is important that the population is informed about the risk factors for this disease and how to prevent it. The aim of this study is to present the oral lesions diagnosed in the oral cancer prevention campaign in the city of Fernandópolis/SP in 2017. Methodology: Initially, patients were examined by dental surgeons at basic health units and, in the presence of oral lesions, they were referred to the Center for Dental Specialties (CEO) of the municipality for reevaluation by a specialist. Results: 720 people were examined during the oral cancer prevention campaign in the city of Fernandópolis / SP in 2017, 58 (8.05\%) of whom had oral lesions and were referred for reassessment by the CEO. Only 52 attended the scheduled appointment, that is, $6(10.3 \%)$ dropped out. Of the re-evaluated patients, $3(5.8 \%)$ presented malignant lesions and needed to be referred for specialized treatment. The other reevaluated patients had 47 benign or potentially malignable lesions and 19 variations in normality were diagnosed. Conclusions: Given the results obtained, we could conclude that the oral cancer prevention campaign provided the diagnosis of many oral lesions even though it did not have the necessary dimension, because only a small portion of the population of the municipality was evaluated during the campaign.
\end{abstract}

Keywords: Collective health; Oral cancer; Dentistry.

\title{
Resumen
}

Introducción: La odontología tiene un gran reto frente a la lucha contra el cáncer de boca, ya que se considera un problema de salud pública. Por ello, es importante que la población esté informada sobre los factores de riesgo de esta enfermedad y cómo prevenirla. El objetivo de este estudio es presentar las lesiones orales diagnosticadas en la campaña de prevención del cáncer oral en el municipio de Fernandópolis/SP en 2017. Metodología: Inicialmente, los pacientes fueron examinados por los cirujanos dentistas de las unidades básicas de salud y, en presencia de lesiones orales, fueron referidos al Centro de Especialidades Odontológicas (CEO) del municipio para reevaluación por un especialista. Resultados: 720 personas fueron examinadas durante la campaña de prevención del cáncer bucal en la ciudad de Fernandópolis / SP en 2017, de las cuales 58 (8.05\%) tenían lesiones bucales y fueron remitidas para reevaluación por parte del CEO. Solo 52 acudieron a la cita programada, es decir, $6(10,3 \%)$ abandonaron. De los pacientes reevaluados, $3(5,8 \%)$ presentaron lesiones malignas y precisaron ser referidos para tratamiento especializado. Los otros pacientes reevaluados tenían 47 lesiones benignas o potencialmente malignas y se diagnosticaron 19 variaciones en la normalidad. Conclusiones: A la vista de los resultados obtenidos, podríamos concluir que la campaña de prevención del cáncer oral proporcionó el diagnóstico de un gran número de lesiones orales aunque no tuvo la dimensión necesaria porque sólo una pequeña parte de la población del municipio fue evaluada durante la campaña.

Palabras clave: Salud colectiva; Cáncer oral; Odontología.

\section{Introdução}

Apesar do grande avanço da Medicina, o câncer bucal ainda é um grande problema de saúde pública. Suas características clínicas são amplamente conhecidas, mas seu diagnóstico, na grande maioria, permanece sendo tardio. (Ribeiro, Martins \& Fernandes, et. al, 2008)

Exames complementares como a biópsia continua sendo o meio mais eficaz para que seja realizado o diagnóstico definitivo da doença. No entanto, existem métodos auxiliares, como a fluorescência óptica que podem favorecer o diagnóstico precoce do câncer bucal. (Tomo, Miyahara \& Simonato, 2019)

Cada vez mais é fundamental que cirurgiões-dentistas estejam capacitados para realizar um exame clínico detalhado dos seus pacientes para que estes possam ser encaminhamos precocemente para os tratamentos necessários, oncológicos ou não, a fim de evitar que as taxas de mortalidade e morbidade aumentem. (Torres-Pereira, Angelim-Dias \& Melo et al., 2012)

Segundo dados divulgados pelo Instituto Nacional de Câncer (INCA), no Brasil, cerca de 15 mil casos de câncer de boca são diagnosticados por ano. O fumo e o consumo exagerado de bebidas alcoólicas são os principais fatores de risco, além da radiação solar e a infecção viral pelo papilomavirus humano (HPV). Homens aparecem com maior predileção, em especial, na idade adulta. (Inca, 2019) 
As campanhas de saúde bucal são fundamentais para abordagens coletivas, prevenção e detecção precoce, orientação e esclarecimento sobre higiene bucal. Em cada campanha é utilizado um método para atingir o máximo de pessoas possíveis, a fim de alertar sobre doenças e, também, orientar sobre questões simples como escolha da escova e do fio dental. A proposta desse tipo de atividade é beneficiar a comunidade, instituir a filosofia preventiva e promover ações educativas induzindo mudanças de hábitos. (Da Costa, Torres, Fonseca, Wada \& De Sousa, 2013)

O objetivo deste trabalho é apresentar as lesões bucais diagnosticadas na campanha de prevenção do câncer bucal no município de Fernandópolis/SP no ano de 2017.

\section{Metodologia}

Neste estudo prospectivo, a captação dos pacientes da amostra foi realizada na rede de atenção à saúde do município de Fernandópolis/SP durante a campanha de prevenção do câncer bucal no ano de 2017. O recrutamento dos pacientes foi realizado por meios de comunicação em massa (rádio, jornal, televisão e internet) e por meios alternativos (folder, banner e faixa). Os agentes de saúde comunitárias reforçaram o convite e orientaram aqueles que apresentavam interesse em participar da campanha.

Todos os pacientes e responsáveis que estiveram de acordo em participar voluntariamente da pesquisa assinaram o Termo de Consentimento Livre e Esclarecido.

Os exames intrabucais foram realizados nas unidades básicas de saúde (UBS) de Fernandópolis/SP de forma sistemática em todas as estruturas anatômicas da boca e orofaringe por meio de inspeção visual e palpação. Os pacientes que apresentaram alguma alteração em tecidos moles foram encaminhados para reavaliação com um profissional especialista no Centro de Especialidades Odontológicas (CEO) no município, onde, se necessário, exames complementares foram solicitados e/ou realizados para a definição do diagnóstico.

O tratamento instituído foi determinado de acordo com laudo do exame anatomopatológico, podendo ser o acompanhamento clínico, tratamento in loco ou o encaminhamento para serviço de saúde terciário no caso diagnóstico de neoplasia maligna.

\section{Resultados e Discussão}

Durante a campanha de prevenção do câncer bucal no ano de 2017, foram examinadas 720 pessoas, sendo que 58 $(8,05 \%)$ dessas apresentaram lesões bucais e foram encaminhadas para reavaliação no CEO. Apenas 52 compareceram a consulta agendada, ou seja, 6 (10,3\%) pessoas evadiram. Dos pacientes reavaliados, 3 (5,8\%) apresentaram lesões de caráter maligno e precisaram ser encaminhados para tratamento especializado. Os demais pacientes reavaliados apresentaram 47 lesões benignas ou potencialmente malignizáveis e 19 variações da normalidade foram diagnosticadas.

Com frequência o profissional a posterga a realização da biópsia quando a lesão não possui características clínicas suspeitas, fato que é bastante comum em lesões em estágio inicial. Além disso, precocemente, as lesões, na sua maioria, são assintomáticas e podem nunca serem notadas pelo paciente, por isso a importância do cirurgião-dentista, do autoexame e das campanhas de prevenção. (Biazevic, Castellanos, Antunes, Michel-Crosato \& Trends, 2006)

A Organização Mundial da Saúde (OMS) desde 2005 utiliza o termo "lesões potencialmente malignas" para descrever lesões ou condições que possuem maior potencial de malignidade. São lesões benignas, porém, quando associadas à fatores de risco podem ou não malignizar-se. As lesões potencialmente malignas são as leucoplasias, as eritroplasias, o líquen plano e a queilite actínica. As condições potencialmente malignas são as fibroses submucosas, o xeroderma pigmentoso e a anemia de Fanconi. (Inca, 2011) 
As lesões benignas diagnosticadas na campanha de prevenção do câncer bucal de Fernandópolis/SP, em 2017, foram citadas na Tabela 1, dentre a leucoplasia, o líquen plano e a queilite actínica, que são classificadas como potencialmente malignas.

O termo leucoplasia foi utilizado pela primeira vez, segundo Grinspan (1973), por Schwmer (1877), para caracterizar lesões brancas da mucosa bucal sem causa definida. É a lesão potencialmente maligna mais frequente da cavidade oral, podendo se desenvolver em qualquer região, porém, as áreas mais acometidas são: mucosa jugal, lábio inferior e língua. Sua etiologia está relacionada ao tabagismo e tem predileção pelo sexo masculino, especialmente, em pacientes de meia idade. Seu diagnóstico é complexo na maioria das vezes, pois há ausência de sintomas.

O líquen plano é uma doença autoimune, mediada por linfócitos T e possui etiologia é desconhecida, no entanto, tem sido associado a fatores psicossociais e doenças sistêmicas. Afeta o epitélio escamoso estratificado, geralmente, acomete mucosa oral, mas pode envolver pele, unhas e mucosa genital. É comum em mulheres de meia idade, em proporção de 3:2 em relação à homens. (Neville, Damm, Allen \& Bouquot, 2004) (Eisen,1999)

Para seu diagnóstico é necessário que sejam realizados tanto exame clínico quanto exame histológico, pois as aparências clínicas do líquen plano variam muito, fazendo com que a doença possa ser negligenciada durante o exame clínico do paciente. Entre os exames complementares, o mais importante é a imunofluorescência direta, que auxilia em casos que possam se assemelhar a outras doenças. (Neville, Damm, Allen \& Bouquot, 2004) (Sano, Quarracino \& Águas, 2005)

A queilite actínica é uma lesão potencialmente maligna que afeta, principalmente, o lábio inferior de pessoas sujeitas a exposição solar, sendo homens da raça branca com idade entre 40 a 80 o grupo mais acometido. Tem progressão lenta e apresenta características de lábios secos, rachados, esbranquiçados ou com placas queratóticas. (Carvalho, de Moraes, Lemos, Santiago Júnior, Vasconcelos \& Pellizzer, 2019) A seleção da sua terapia deve ser guiada por achados histopatológicos, exame clínico e exames de rotina. O diagnóstico precoce e a prevenção são extremamente importantes nessas doenças. (Lai, Pampena, Cornacchia, Pellacani, Peris \& Longo, 2019)

Tabela 1: Lesões benignas diagnosticadas durante a Campanha de Prevenção ao Câncer Bucal, Fernandópolis, SP, 2017.

\begin{tabular}{|c|c|}
\hline Lesões benignas & N $^{\circ}$ \\
\hline Fibroma & 9 \\
\hline Candidíase & 7 \\
\hline Leucoplasia & 6 \\
\hline Úlcera aftosa recorrente & 6 \\
\hline Hemangioma & 4 \\
\hline Hiperplasia fibrosa inflamatória & 3 \\
\hline Nevus & 3 \\
\hline Papiloma & 3 \\
\hline Queilite actínica & 2 \\
\hline Tatuagem por amálgama & 2 \\
\hline Líquen plano & 1 \\
\hline Síndrome da ardência bucal & 1 \\
\hline TOTAL & 47 \\
\hline
\end{tabular}

*Dados fornecidos pelo estudo original. Fonte: Autores.

As variações da normalidade não apresentam necessariamente caráter de doença, porém, não exime do profissional responsável pelo exame clínico, o esclarecimento e a orientação do paciente que apresentar qualquer alteração em meio bucal. São relativamente comuns e apresentam, em muitos casos, caráter genético, tendo frequência aumentada conforme a idade. Na 
campanha de prevenção do câncer bucal de Fernandópolis/SP, em 2017, foram diagnosticadas as variações da normalidade conforme demostrado na Tabela 2.

Tabela 2: Variações da normalidade diagnosticadas durante a Campanha de Prevenção ao Câncer Bucal, Fernandópolis, SP, 2017.

\begin{tabular}{|c|c|}
\hline Variações da Normalidade & No $^{\circ}$ \\
\hline Varicosidades linguais & 1 \\
\hline Língua geográfica & 3 \\
\hline Tórus (palatino ou mandibular) & 3 \\
\hline Grânulos de Fordyce & 3 \\
\hline Língua fissurada & 6 \\
\hline Pigmentação melânica racial & 3 \\
\hline TOTAL & 19 \\
\hline
\end{tabular}

*Dados fornecidos pelo estudo original. Fonte: Autores.

Chamamos atenção que inúmeras campanhas de saúde bucal são realizadas por todo o Brasil, mas que os principais focos são as doenças periodontais e a doença cárie, esquecendo do câncer de boca. (Antunes, Toporcov \& Wunsch-Filho, 2007) Nesse trabalho, a campanha no município de Fernandópolis teve como foco principal a prevenção de lesões bucais, em especial, o câncer bucal.

A valorização da campanha de vacinação da gripe nos últimos anos ajudou a aumentar a visibilidade da campanha de prevenção e diagnóstico precoce em câncer bucal junto a população, pois as duas ocorrem simultaneamente demostrando o esforço do Sistema Único de Saúde (SUS) em promover prevenção. (Da Costa, Torres, Fonseca, Wada \& De Souza, 2013) No município de Fernandópolis/SP, o exame intrabucal foi realizado logo após o paciente ser imunizado abrangendo, em especial, idosos, ou seja, pessoas a partir de 60 anos, já que é considerado o grupo de risco o câncer de boca.

Horowitz et al. (1998) afirmaram que uma das necessidades para diminuir a quantidade de indivíduos predispostos ao câncer bucal, é dar enfoque a educação populacional sobre os fatores de riscos. Dada a natureza comportamental dos fatores de risco envolvidos com o câncer bucal, a abordagem deve ser multiprofissional.

A boca está em contato direto com o tabaco, o que facilita a ação de elementos cancerígenos. O tabaco associado ao álcool provoca sinergismo aumentando mais a possibilidade do aparecimento do câncer bucal. O etilismo constitui uma síndrome multifatorial, comprometimento de origem física e mental, além dos impactos sociais. É considerado o segundo fator causador do câncer de boca, principalmente, de língua e assoalho bucal. A radiação solar, em longo prazo, apresenta significativa importância biológica e se torna um considerável fator de risco. (Horowitz, et al., 1998)

É importante cada vez mais a atualização e o esclarecimento dos cirurgiões-dentistas em relação a doença, a fim de capacitar o profissional ao diagnóstico precoce. O treinamento dos profissionais de saúde é uma etapa fundamental nas campanhas, podendo melhorar seus resultados. (Da Costa, Torres, Fonseca, Wada \& De Sousa, 2013) O treinamento dos cirurgiões-dentistas da rede de atenção à saúde do município de Fernandópolis/SP ocorreu com a participação de profissional especialista em diagnóstico bucal.

A intenção dessas campanhas é identificar casos ainda em fase inicial, para que, as intervenções terapêuticas possam ser benéficas, tanto em termos de sobrevida quanto de qualidade de vida e assim proporcionar um melhor prognóstico ao paciente. (Ribeiro, Martins, Fernandes et al., 2008) O diagnóstico tardio explica o alto índice de mortalidade. A falta de informação, tanto do profissional quanto do paciente, aliado a precariedade ao acesso às consultas odontológicas e ao exame clínico realizado corretamente. Por isso, as campanhas de prevenção são tão importantes para que a informação chegue na maior quantidade de 
pessoas possíveis. As campanhas tem como meta a conscientização da população a importância dos fatores de risco e, também, do diagnóstico precoce, para que assim, seja possível proporcional um prognóstico favorável. (Kowalski, Magin Riera, Coelho \& Zeferino, 1991) (Antunes, Toporcov \& Wunsch-Filho, 1993)

Normalmente, essas campanhas envolvem apenas a realização do exame físico visual, tendo baixos custos para a instituição organizadora. (Almeida, Cazal, Brandão et al., 2005) A divulgação foi realizada em massa, convidando a população a comparecer as UBS no município de Fernandópolis/SP, onde foram realizados exames físico extra e intrabucal, por meio de visualização e palpação das estruturas orofaciais, seguida de anotações em fichas próprias. Os materiais utilizados são de fácil acesso e de baixo custo. Os pacientes que apresentaram lesões foram encaminhados para reavaliação e/ou intervenção especializadas. (Czerninski, Zini, Sgan-Cohen, Silverman, Kerr \& Epstein, 2010)

Ainda Horowitz e colaboradores (1998) afirmaram que mais de $25 \%$ da população não sabe como prevenir o câncer bucal. Patton (2004) relatou que o nível de conhecimento pode ser influenciado pela frequência de contatos educacionais, ou seja, quanto maior o contato com um cirurgião-dentista, maior o conhecimento sobre saúde bucal. Por esse motivo, as pessoas atendidas durante as campanhas realizadas no município de Fernandópolis/SP são mantidas dentro da rede por meio de atendimentos periódicos, seja nas UBS ou no CEO.

\section{Considerações Finais}

Diante dos resultados obtidos, pudemos concluir que a campanha de prevenção do câncer de boca do município de Fernandópolis/SP no ano de 2017 proporcionou o diagnóstico de um número grande de lesões bucais mesmo não tendo a dimensão necessária, pois apenas uma pequena parcela da população do município foi avaliada durante a campanha. Com isso, nossos planejamentos foram realizados para que motive profissionais e população para sua continuidade e ampliação;

\section{Referências}

Almeida, F. C. S., Cazal, F., Brandão, T. B. et al. (2005). Campanha de popularização do auto-exame da boca - Universidade de São Paulo, Brasil (Parte I). Rev. Bras. Patol. Oral. 2005; 4 (3), 147-56. http://revodonto.bvsalud.org/pdf/rbo/v70n2/a07v70n2.pdf

Antunes, J. L. F, Toporcov, T. N, \& Wunsch-Filho, V. (2007). Resolutividade da campanha de prevenção e diagnóstico precoce do câncer bucal em São Paulo, Brasil. Revista Panam Salude Publica. (2007) 21(1):30-6. https://iris.paho.org/bitstream/handle/10665.2/7895/a04v21n1.pdf?sequence=1\&isAllowed=y

Armelin, A. M. L., Hernandes, A. P., Tomo, S. Miyahara, G. I., Marques. T. C. N, \& Simonato, L. E. (2019). Oral lesions detected durind a populaton screening for prevention and Early diagnosis of oral câncer and potentially malignant disorders. RFO UPF, Passo Fundo. (2019); 350-359, 10.5335/rfo.v24i3.9818

Axéll, T. (1987). Ocurrence of leukoplakia and some other oral white lesions among 20, 333 adult Swedish people. Communit Dent Oral Epidemio. (1987), Feb. 46-51. 10.5935/1676-2444.20140008

Biazevic, M. G., Castellanos, R. A., Antunes, J. L., \& Michel, C. E. (2006). Trends in oral cancer mortality in the city of São Paulo, Brazil. Cad. Saude Publica. (2006); 22(10):2105-14, 10.1590/s0102-311x2006001000016

Carrer, F. C. A (2019). SUS e Sáude Bucal no Brasil: Por um future com motivos para sorrir. Faculdade de Odontologia USP. (2019) 614-0981 978-85-7040018-5 http://www.fo.usp.br/wp-content/uploads/2019/01/SUS-e-a-Sa\%C3\%BAde-Bucal-no-Brasil.pdf

Carvalho, M. V, De Moraes, S. L. S., Lemos, C. A. A., Santiago, J. J. F., Vasconcelos, B. C. D. E., \& Pellizzer, E. P. (2019). Surgical versus non-surgical treatment of actinic cheiltins: a systematic review and meta-analysis. Oral dis. (2019) May;25(4):972-81. 10.1111/odi.12916

Czerninski, R., Zini, A., \& Sgan-Cohen, H. D. (2010). Lip cancer: indidence, trends, histology and survival. British Journal Dermatol. (2010) 1624:1103-9. Doi 10.1111/j.1365-2133.2010.09698.x

Da Costa, A. M., Torres, L. H. N., Fonseca, D. A. V., Wada, R. S., \& De Sousa, M. R. (2013). Campanha de prevenção e diagnóstico precoce do câncer bucal: perfil dos idosos participantes. Rev. Bras. Odontol. (2013). http://revodonto.bvsalud.org/pdf/rbo/v70n2/a07v70n2.pdf

Eisen, D. (1999). The evaluation of Cutaneous, genital, scalp, nail, esophageal and ocular involviment in patients with oral lichen planus. Oral Surg Oral Med Pathol Radiol Endod. (1999). 88:431-6. 10.1016/s1079-2104(99)70057-0

Grispan, D. (1973). Enfermidades de la boca,Tomo II. Patologia clínica y terapêutica de mucosa bucal. Mundi Buenos Aires. (1973).123-325.

Horowitz, A. M., Moon, H. S., Goodman, H. S, et. al. (1998). Maryland adults knowledge of oral câncer and having oral câncer examinations. Journal of Public Health Dentistry. (1998) 58(4):281-7. doi 10.1111/j.1752-7325.1998.tb03010.x 
Ismail, S. B, Kumar, S. K. S, \& Zain, R. B. (2007). Oral lichen planus and lichenoid reactions: etiopathogenesis, diagnosis, management and malignant transformantion. Journal of the American Academy of Dermatology. (2007) 49:89-106 doi 10.2334/josnusd.49.89

Lai, M. P. R. Cornacchia, L. Pellacani, G. Peris, K, \& Longo. C. (2019) Treatmens of actnic cheilitis: a systematic review of the literature. Journal of the American Academy Of Dermatology. (2019) 25(3):876-887. doi 10.1016/j.jaad.2019.07.106

Martins, J. S, Abreu, S. C. C, Araújo, M. E, Bourget, M. M. M, Campos, F. L, Grigoletto, M. V. D, et al. (2012). Estratégias e resultados da prevenção do câncer bucal em idosos de São Paulo, Brasil, Rev Panam Salud Publica. (2012) 31(3):246-52. https://iris.paho.org/bitstream/handle/10665.2/9346/10.pdf?sequence=1

Ministério da Saúde. Instituto Nacional do Câncer. (2013) Uma grande contribuição do Brasil sorridente foi a portaria874/2013 https://www.inca.gov.br/publicacoes/legislacao/portaria-874-16-maio-2013

Ministério da Saúde. Instituo Nacional do Câncer. (2021). Tipos do câncer de boca. https://www.inca.gov.br/tipos-de-cancer/cancer-de-boca

Ministério da Saúde, Instituo Nacional do Câncer. (2013). Coordenação de prevenção e vigilância, estimativa incidência de câncer no Brasil. HTTP://WWW.INCA.GOV.BR/ESTIMATIVA/2012.

Ministério da Saúde. Instituo Nacional do Câncer (2009). Inquérito domiciliar sobre comportamentos de risco e morbidade referida de doenças e ag ravos não transmissíveis: Brasil, 15 capitais e Distrito Federal, 2002-2003. HTTP://WWW.INCA.GOV.BR/INQUERITO/DOCS/COMPLETA.PDF

Ministério da Saúde. Instituo Nacional do Câncer. (2019) Semana da saúde bucal e alerta ara cuidados contra o câncer de boca. HTTPS://WWW.INCA.GOV.BR/NOTICIAS/SEMANA-DA-SAUDE-BUCAL-E-ALERTA-PARA-CUIDADOS-CONTRA-O-CANCER-DE-BOCA

Neville, B. W, Damn, D. D, Allen, C. M, \& Bouquot, J. E. (2004). Patologia oral e Maxilofacil 2ed. Rio de Janeiro: Guanabara Koogan S.A: 2004. (pp:559639)

Patton, L.L, Agans, R., Elten, J. R, et al. (2004). Oral câncer knowledge and examination experiences among noth carolina adults. Journal Publ. Health Dent. (2004) 64 (3): 73. 10.1111/j.1752-7325.2004.tb02748.x

Ribeiro, R. Martins, M. A. T, Fernandes, K. P. S, et.al. (2008) Avaliação do nível de conhecimento de uma população envolvendo câncer poral. Robranc. (2008) 17(44):104-9 https://www.robrac.org.br/seer/index.php/ROBRAC/article/view/40/30

Sano, S. M. Quarracino, M. C., Aguas, S. C., González, E. J., Harada, L. Krupitzki, H, \& Mordoh, A. (2008) Sensitivity of direct immunofluorescence in oral diseases. Study of 125 cases. Med Oral Patol Oral $\quad$ Cir $\quad$ Bucal. http://www.medicinaoral.com/medoralfree01/v13i5/medoralv13i5p287.pdf

Schepman, K. P, Van Der, E. H, Smeele, L. E, et al. (1998) Malignant transformation of oral leukoplakia: a follow-up study of a hospital-based population of 166 patients with oral leukoplakia from the Netherlands. Journal Oral Oncology (1998). 34(4):270-5. 10.1016/S1368-8375(98)80007-9

Silverman, J. R. S. Kerr, A. R, \& Epstein, J. B. (2010) Oral and pharyngeal cancer control and early detection. Journal Cancer Educacional. (2010) 25:279-81. $10.1007 / \mathrm{s} 13187-010-0045-6$

Tomo, S. Miyahara, G. I. \& Simonato, L. E. (2019). History and future perspectives for the use of fluorescence visualization to detect oral squamous cell carcinoma and oral potentially malignant disorders. Journal Pre-proof Photodiagnosis and Photodynamic Therapy. (2019) 308-317; 10.1016/j.pdpdt.2019.10.005

Torres-Pereira, C. C., Angelim, D.A. Melo, N. S, et, al. (2012); Abordagem do cancer da boca: uma estratégia para os níveis primário e secundário de atenção em sáude. Cad. Saúde. Pública. (2012) 28:S30-S39 doi 10.1590/S0102-311X2012001300005 